\title{
Introduction to Confocal Microscopy
}

Judith A. Drazba

Imaging Core, NB10, Cleveland Clinic, 9500 Euclid Avenue, Cleveland, OH 44195

Statement of the Problem and Early Development Theorizing in 1957 about how brains learn, Marvin Minsky wanted to see how neuronal cells connect. He had brightfield and electron microscopy at his disposal but neither could produce the desired result. Brightfield images appear out-of-focus due to increasing light scattering as the specimen thickness increases. Electron microscopy produces excellent in-focus images of multiple layers of a sample but only after the sample is mechanically cut into those layers and thus destroyed. Even then, the result is a series of two-dimensional images rather than a three-dimensional representation of the intact specimen. Minsky worked on an imaging technique proposed by Paul Nipkow [a German physicist and television pioneer] and built a "confocal imaging" microscope to solve this problem. However, it took others like Amos and White in 1987- and the advent of improved lasers, computers, and microelectronics - for this invention to become practical.

What's Unique About a Confocal These instruments have several advantages over conventional microscopes. They reject out-of-focus light to produce images of higher resolution. This is accomplished by introducing physical pinholes into the light path. The pinhole on the excitation side allows light to illuminate only a small region of the specimen. (Most systems using lasers as their light source forego this pinhole because the laser is already a tightly focused beam.) But even some of this focused light will be scattered by other points in the imaging plane. These errant rays can be rejected by placing a second pinhole just before the detector. Combined with precise controls and robust computers, this visual clarity allows confocals to optically slice through a specimen with little harm and produce a real-life 3D representation from either a fixed or live sample.

Applications Confocals work best where out-of-focus light is dense. Thus, samples do not need to be thin. With a low magnification dry lens $(10 \mathrm{X})$ it is possible to optically section several mm into transparent tissue. At higher magnification $(63 \mathrm{X})$ with an opaque specimen the limit may be closer to $30 \mu \mathrm{m}$. The specimen must either be reflective or stained with a fluorescent label, e.g., antibodies or dyes that specifically recognize and bind to cellular components (organelles, genes, proteins). This can pose a problem with thick specimens where diffusion of the label into the sample is a major limiting factor. Using detergents and increased incubation times can help. The best samples are those in which cells have been genetically engineered to endogenously produce fluorescent proteins such as GFP. Today specimens are routinely tagged with combinations of labels to enable investigators to see relationships between different structures in a single sample. Numerous fluorescent physiologic indicators are available that penetrate living cells and allow the confocal to be used in 4D to analyze changes in ion concentrations, $\mathrm{pH}$ gradients, and membrane potentials.

Operational Considerations To obtain images that most accurately represent the sample attention must be paid to several issues: 1) Lenses should have high numerical aperture and be corrected for chromatic aberration. Use a planapochromat lens if possible. 2) Avoid refractive index mismatch between the specimen mounting medium and the objective immersion medium that will result in geometric distortion in the z-axis. 3) Optimize pinhole size. The best resolution/signal compromise 
is reached when the size of the pinhole equals the size of the Airy disk of the image. 4) The relationship between step size and optical section thickness will determine the quality of the subsequent 3D reconstruction.

Beyond Confocal Two major drawbacks to confocal imaging are a) the short distance that visible light can penetrate into a specimen and b) photobleaching of the entire specimen while collecting information from a single optical plane. Multiphoton microscopy helps solve these problems. While it uses the same basic hardware as a confocal (companies sell instruments that are combination confocal and MP systems), it employs long wavelength lasers and there is no need for pinholes. The longer wavelength light is able to penetrate more deeply into specimens and any photobleaching occurs only in the plane of focus.

Reference: $\underline{\text { http://microscopy.fsu.edu }}$

\section{WIDE-FIELD}

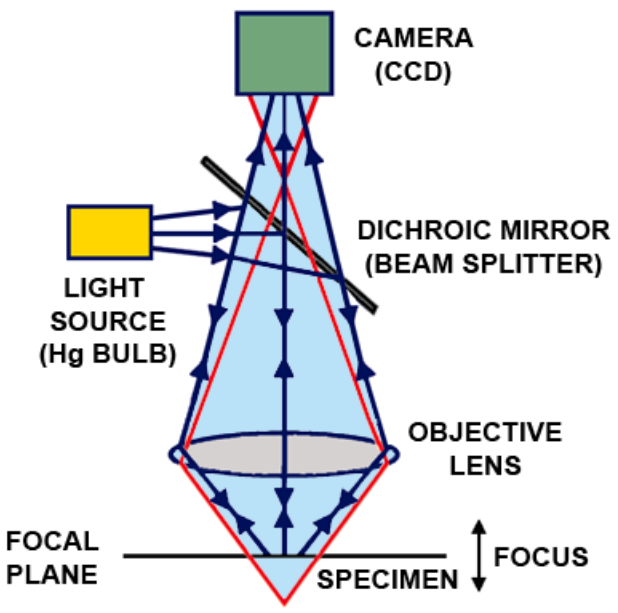

- IN-FOCUS LIGHT RAYS
- OUT-OF-FOCUS LIGHT RAYS
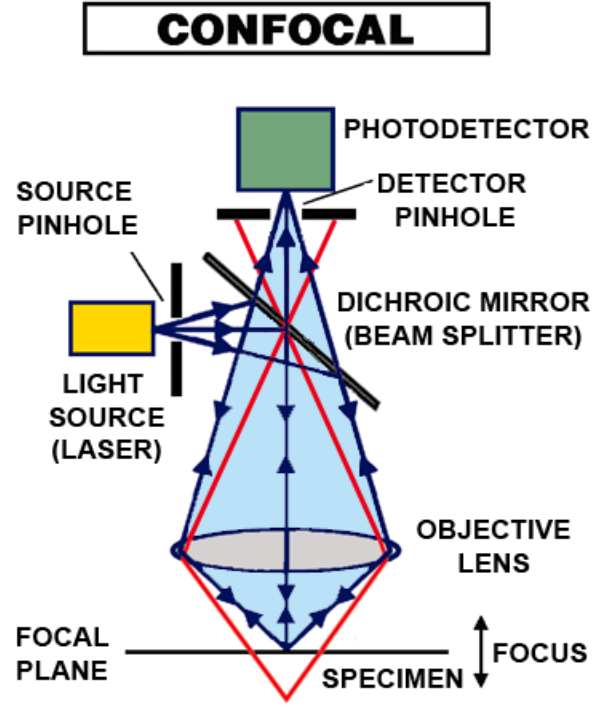

- IN-FOCUS LIGHT RAYS

- OUT-OF-FOCUS LIGHT RAYS

Figure 1. Comparison of the light path of a wide-field and a confocal microscope
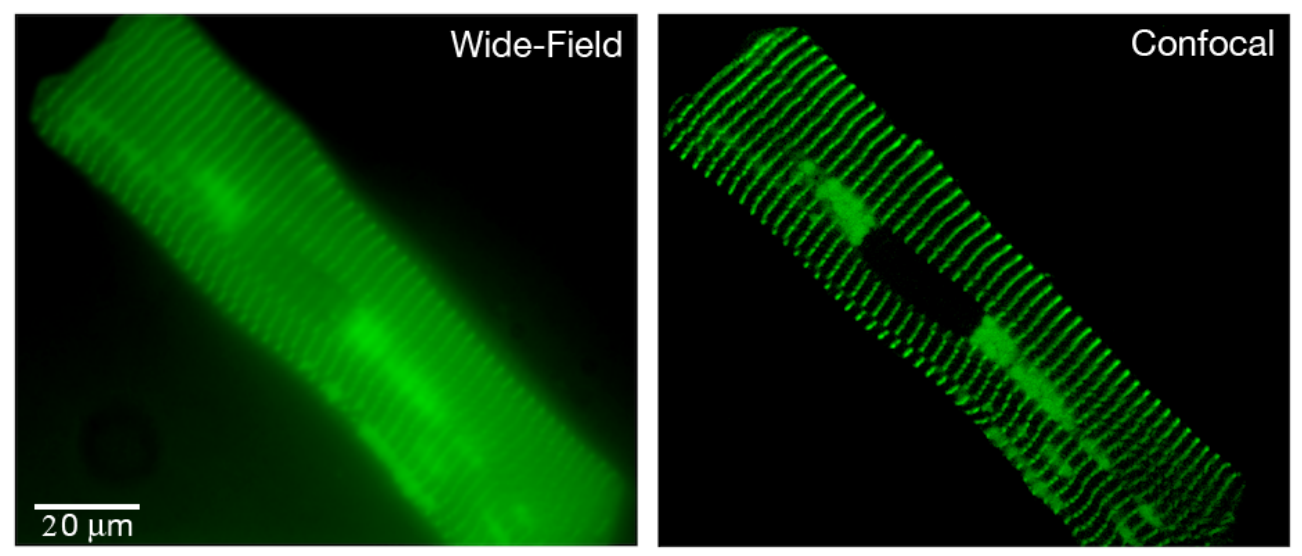

Figure 2. Comparison of $\alpha$-actinin-stained cardiac myocyte images collected from the same sample on both wide-field and confocal microscopes. Note the improved detail of the confocal image. 